\title{
Exercise as a prescription therapy for breast and colon cancer survivors
}

This article was published in the following Dove Press journal:

International Journal of General Medicine

15 April 2013

Number of times this article has been viewed

\author{
Giorgio Galanti' \\ Laura Stefani' \\ Gianfranco Gensini² \\ 'Sports Medicine Center, \\ ${ }^{2}$ Department of Medical and \\ Surgical Critical Care, University \\ of Florence, Florence, Italy
}

Correspondence: Laura Stefani Sports Medicine Center, Viale Morgagni 85, University of Florence,

Florence 50100 , Italy

Tel +390557947066

Fax +3905 57947066

Email laura.stefani@aouc.unifi.it
Background: Breast and colon tumors are the most common types of cancer in the general population. As a result of improved diagnosis and treatment, more people are now surviving cancer. Lifestyle has been identified as one of the potential risk factors for cancer, and it has been demonstrated recently that physical activity reduces the physiological and psychological symptoms and side effects of chemotherapy. Muscular atrophy, weight changes, decreased aerobic capacity, fatigue, and depression are the most common symptoms in cancer patients. The exact amount of moderate to vigorous physical activity needed to overcome these effects has not yet been established, but an individualized and variable exercise program as prescription therapy could be considered a helpful tool for improving quality of life. This paper proposes a model of lifestyle analysis and dedicated individualized exercise programs for the treatment of cancer patients. The program starts with a preliminary evaluation of the patient's lifestyle using a questionnaire and accelerometer, which provides information on the amount of daily physical activity and number of steps taken, as well as an assessment of aerobic capacity, cardiovascular response, muscle strength, and resistance. Two different levels of aerobic exercise, ie, low-to-moderate ( $40 \%$ of maximal heart rate) to moderate $(60 \%$ of maximal heart rate) can be prescribed. This model of exercise prescription in patients with cancer offers a flexible program for assisting in the management of this complex disease. It is projected to ensure easier management of medical problems related to cancer, and to attract a greater number of participants over time.

Keywords: physical exercise, therapy, breast cancer, colon cancer, lifestyle

\section{Introduction}

Cancer is the second leading cause of death, with the most frequently diagnosed cancers in the general population being breast and colon tumors. ${ }^{1}$ In addition to genetic factors, diet and lifestyle, including smoking, alcohol consumption, and sedentarism have been identified as potential risk factors. ${ }^{2-4}$ Recent studies have demonstrated the beneficial effects of regular physical activity in reducing the global incidence of cancer, as well as reducing psychological problems and helping to control the consequences of disease progression. ${ }^{5,6}$ In particular, muscular atrophy, weight changes, decreased aerobic capacity, fatigue, depression, and reduced muscle strength and flexibility have a significant impact on quality of life for patients with progressive disease. ${ }^{7,8}$ Studies have demonstrated that the majority of these side effects are controlled by regular physical activity. ${ }^{9}$ The authors have focused on the relevance of a prospective surveillance model for rehabilitation of women with breast cancer, with intervention to promote and support physical activity throughout the course of treatment. ${ }^{10,11}$ 
In parallel, a systematic literature review has highlighted the importance of distinguishing between "physical activity", which includes any movement of the skeletal muscles, and "exercise", which is a specific type of physical activity for improving and/or maintaining physical fitness. ${ }^{12}$

In fact, aerobic and strengthening physical exercises are now considered to be important tools in the treatment of cancer, not only for rehabilitation of patients, but also for decreasing potential lifestyle risk factors for cancer which are closely related to progression of the disease. It has been established that a "moderate amount of daily aerobic physical activity" in the range of $65 \%-80 \%$ of maximal heart rate can help to improve health in the general population. ${ }^{13}$ Moreover, the current US literature has recently addressed "exercise as prescription therapy in cancer survivors", highlighting the beneficial effects of exercise at different levels of intensity. ${ }^{14,15}$

Further studies ${ }^{16,17}$ have investigated other aspects of exercise as a prescription therapy, and demonstrated that physical exercise needs to be supervised and rigorously followed. The literature has not yet clarified the outcomes of long-term exercise therapy beyond a 10-week duration, during which participation and adherence with physical exercise is important. ${ }^{18}$ This paper describes an approach to physical exercise for therapeutic purposes and to improve the quality of life for patients with breast or colon cancer, which are among the most common malignancies and may have a longer life expectancy. This program includes survivors of breast and colon cancer, without metastatic involvement, who can undertake unsupervised physical exercise in the home environment, with the expectation that they can achieve both subjective and objective improvement in their quality of life. Such improvements may be possible, even with regular moderate exercise, and may lessen the consequences of comorbidity. The choice of two different levels of exercise should also increase adherence with the program, both qualitatively and quantitatively.

\section{Study design}

In order to highlight the efficacy and empowerment achieved by the exercise program, this protocol proposes selection of two main subgroups of cancer survivors, ie, those able to perform low-to-moderate or moderate exercise. Inclusion of these two levels of exercise intensity in the program should also encourage participation by a greater number of patients, and offers the possibility of modulating the level of exercise as therapy according to disease severity. The aim of this strategy is not only to achieve an immediate therapeutic effect on quality of life in cancer patients, but also to identify a model which can be adapted to provide different levels of exercise tailored to the individual in order to have the greatest benefit. It would also be very important to maintain maximum patient participation. Sports trainers, experienced in the use of common physical exercise models, will be helpful in implementation of this therapeutic approach by providing optimal supervision of physical exercise in cancer survivors. They will also have a significant role in periodic evaluation of patients for their biomechanical status. The psychological aspects of quality of life in cancer patients will also be assessed and monitored for several variables, including anxiety, depression, confusion, energy, and anger during follow-up using Linear Analog Self Assessment scores. ${ }^{19}$

\section{Practical aspects of the program}

Two groups of cancer survivors, ie, those with breast or colon cancer without metastasis, will be considered for the "exercise prescription" program at our sports medicine unit, and will be enrolled after assessment in the oncology unit. Verbal and written consent will be obtained from each patient. Only subjects in otherwise good physical condition, without anemia or cardiovascular, pulmonary, thromboembolic complications, or other important comorbidities, will be included. All patients will undergo a complete anamnesis and clinical checkup. Clinical evaluation at the sport medicine unit will include assessment of hemodynamic parameters related to exercise tolerance in two separate sessions, ie, at the first appointment and 7 days later. In schematic terms, the baseline assessment includes taking of a detailed medical history, an electrocardiogram, an echocardiogram, lifestyle analysis by questionnaire, six-minute walking test (6MWT) with assessment of blood pressure at rest and at peak effort, and measurement of heart rate and respiratory rate using recording leads placed on the patient's chest. Assessment of body water balance will be performed using bioimpedance analysis. At the end of this session, a food diary is provided and an accelerometer (AiperMotion 500 PC, Aipermon $\mathrm{GmbH} \& \mathrm{Co}$, Munich, Germany) for monitoring of daily motor activity is positioned on the patient's waist. The patient is instructed to wear the accelerometer device only during the day and to return with it 7 days later. This device is equipped with an oscillometer which measures acceleration of body movement along axes of spatial reference. The calculations are based on the principle of inertia of body/mass when subjected to acceleration $(\mathrm{F}=\mathrm{m} * \mathrm{a})$ along one or more axes, combined with specific algorithms (pedometers, calculating calories, 
distance, steps) designed to correlate with acceleration of body movement, energy consumed by steps and distance traveled, and type of activity (ie, fast, slow, or passive). The accelerometer calculates the amount of spontaneous and programmed daily physical activity, from which it is possible to obtain useful information on the daily level of spontaneous motor physical activity and the amount and intensity of programmed motor activity, which may include walking, slow jogging, or other activities with measurable intensity and energy expenditure.

Motor activity registers are wearable devices which are able to measure body movement, identify elements that characterize motor activity, and correlate this activity with energy consumption, as well as recording, storing, and making the data available for further analysis and evaluation by medical and sports specialists. A recording can be obtained from the accelerometer and used for lifestyle analysis. The results for daily energy expenditure (in kilocalories) obtained from the algorithm include the parameters of age, weight, height, and gender, which are integrated with data obtained from food diary and utilized for calculation of energy balance.

The $6 \mathrm{MWT}^{20}$ can be used as an alternative to the cardiopulmonary test for assessing cardiovascular performance. Although the cardiopulmonary test is generally accepted as the gold standard for estimating the exercise tolerance, the 6MWT may be preferable in the context of cancer survivors. The $6 \mathrm{MWT}$ is an easier method which rapidly identifies the intensity of the exercise prescribed, and if carried out in parallel with Borg's 15-point rating of perceived exertion or 10-point Category Ratio (CR10) scale, it is close to effective exercise perception. The first step on day 1 involves estimation of daily lifestyle habits using a questionnaire ${ }^{21}$ and Karnofsky performance status. ${ }^{22}$ The patient's eating habits will be evaluated by detailed questioning and dedicated software assessing specific aspects of daily nutrition, in particular, the relative contribution of micronutrients and macronutrients, ie, carbohydrates, fats, proteins, saturated fats, antioxidants, vitamins, minerals, fiber, omega 3, and omega 6 fatty acids, together with meal frequency and composition. All anthropometric parameters will be calculated, including body water content. Acquisition of lifestyle data is then completed with information obtained from the accelerometer worn by the subjects for at least 7 days. According to the current literature, lowto-moderate and moderate-to-vigorous aerobic physical activity, corresponds to $40 \%$ and $60 \%$ of maximum effort, respectively, ${ }^{23,24}$ and is normally prescribed on average at least three times a week over about three weeks. The amount and intensity of physical exercise, frequently consisting of fast walking, indoor or outdoor cycling, or any other physical activity, is normally established after cardiopulmonary testing or by the $6 \mathrm{MWT}$.

In addition to distance walked, other parameters collected during the 6MWT can include systolic and diastolic blood pressure at rest and at peak effort, heart rate and rate-perceived exertion both at rest and at the end of the test, and respiratory and heart rates. Calculation of the energy expenditure for estimation of the amount of physical activity (intensity, time, frequency) is done by extrapolation, whereby the heart rate at the end of the 6MWT is considered to correspond to maximum tolerated effort. Using the conversion scale for metabolic equivalents (METs)/24-hour heart rate, calculation of the corresponding METs provides the energy expenditure. An energy expenditure index quantifies the total amount of physical activity performed in a standardized manner across individuals and types of activities, and is calculated as the product of the number of METs associated with one or more physical activities and the number of minutes spent performing those activities (ie, METs per minute). This calculation is usually standardized per week or per day.

\section{Example}

Jogging (at about 7 METs) for 30 minutes three times per week $=7$ METs $\times 30$ minutes $\times$ three times per week $=630$ METs $\times$ minutes $\times$ week. EE $=($ METs $\times \mathrm{kg}) / 60$, where $\mathrm{EE}$ is energy expenditure and 60 represents the minutes in one hour. The MET is an index of energy expenditure, described as "[A MET is] the ratio of the rate of energy expended during an activity to the rate of energy expended at rest. [One] MET is the rate of energy expenditure while sitting at rest by convention, [1 MET is equal to] an oxygen uptake of 3.5 [mLIkgj1 Iminj1]". ${ }^{25}$ Time of exercise $=150 / \mathrm{EE}$, where 150 represents the time currently recognized and recommended for weekly exercise as a prescription therapy.

To take into account possible variations in exercise tolerance in a population for which drug therapy with potential cardiovascular effects, such as beta-blockers or calcium antagonists, is common, the final intensity of exercise is established using a formula which enables the target heart rate to be calculated using a correction scale. Parallel application of the individual perception of effort using the rate-perceived exertion scale (ie, the CR10) ${ }^{26}$ is normally in agreement and confirms these values: $\mathrm{HR}_{\mathrm{t}}=60 \%$ or $80 \% \times\left(\mathrm{HR}_{\max }-\mathrm{HR}_{\text {rest }}\right)+\mathrm{HR}_{\text {rest }}$, where $\mathrm{HR}_{\mathrm{t}}$ is the target 
heart rate, $\mathrm{HR}_{\max }$ is maximum heart rate, and $\mathrm{HR}_{\text {rest }}$ is heart rate at rest.

If the patient prefers to perform aerobic exercise indoors on a treadmill, stationary bicycle, and/or a stair climbing machine, initial supervision by expert trainers can be guaranteed. If outdoor exercise is preferred, it would be necessary to maintain the intensity of exercise previously established. The positive effects of regular exercise on subjective and objective aspects of lifestyle can be estimated from several variables, including anxiety, fatigue, and depression, using the Linear Analog Self Assessment scale, scoring of which allows the clinician to detect improvement in overall performance and to determine if this persists over time.

\section{Analysis of quality of life}

Quality of life is one of the most important considerations for cancer survivors. ${ }^{27,28}$ The American College of Sports Medicine recommendations emphasize the need for patients to be as active as their condition allows. ${ }^{29}$ Lifestyle can be evaluated using a simple questionnaire focusing on the patient's perception of the intensity of their daily physical activity or can be assessed in more detail by patient interview regarding the type of and amount of time spent on physical activity (Figure 1).

Sedentary behavior is defined as an activity that involves limited or no movement, eg, sitting, watching television, playing video games, and using the computer, and has an energy expenditure of about 1-1.5 METs. ${ }^{30}$ Therefore, lifestyle evaluation is done using an accelerometer, the parameters of which allow a more objective distinction between sedentary and active subjects. The accelerometer provides data on medium daily distance covered, with the intensity of physical activity expressed as slow walking (up to $3 \mathrm{~km}$ /hour), fast walking (up to $5 \mathrm{~km} /$ hour), or jogging (up to $7 \mathrm{~km} /$ hour) and also the daily total number of steps taken (Figure 2).

From the accelerometer report, the clinician can identify a sedentary lifestyle from the recorded mean physical activity level, number of daily and weekly steps taken, as well as distance and duration of walking, running, or jogging. These parameters can be compared periodically with data from the patient's previous follow-up appointments.

\section{Analysis of body composition and diet}

Body composition is estimated using several anthropometric parameters, including height, weight, body mass index, waist circumference, hip circumference, and the waist to hip

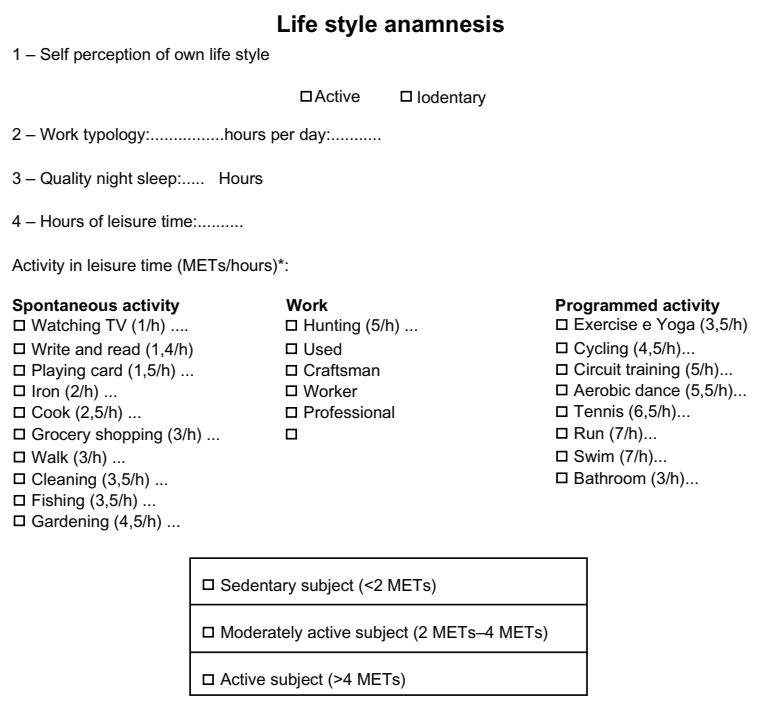

Figure I Questionnaire canvassing daily level of physical activity.

circumference ratio. These data are taken into consideration particularly during the follow-up period. Also included is an analysis of total water distribution, which includes both intracellular and extracellular components, using a bioelectrical impedance vector analysis system (BIA 101, Akern RJL Systems, Florence, Italy). This device also estimates fat mass, free fat mass, and the phase angle (Figure 2). Among the parameters used for analysis of body composition, the phase angle is a potential nutritional indicator used in clinical oncology to follow the trend of patient nutritional status over time, and is advocated for use in advanced colorectal cancer. ${ }^{31}$ Calculation of the phase angle is derived from resistance and reactance values obtained during bioimpedance analysis. Dietary analysis is important in cancer patients for whom increased body weight is often an inevitable outcome of chemotherapy. An increase in fat mass increases cardiovascular risk and is associated with persistence of inflammation, which may increase the risk of disease recurrence. Analysis of the food diaries given to patients in the week prior to the final report often identifies inappropriate food choices in terms of the type and quantity of food eaten. Dietary analysis also includes calculation of the energy balance, ie, the net difference between calorie intake and energy consumption. Accurate dietary analysis can be done using dedicated software which enables calculation of the daily calorie intake.

\section{Biomechanical follow-up}

Biomechanical performance using handgrip for estimation of upper body strength and the 30-second chair stand test for lower body strength is also recorded during the first 

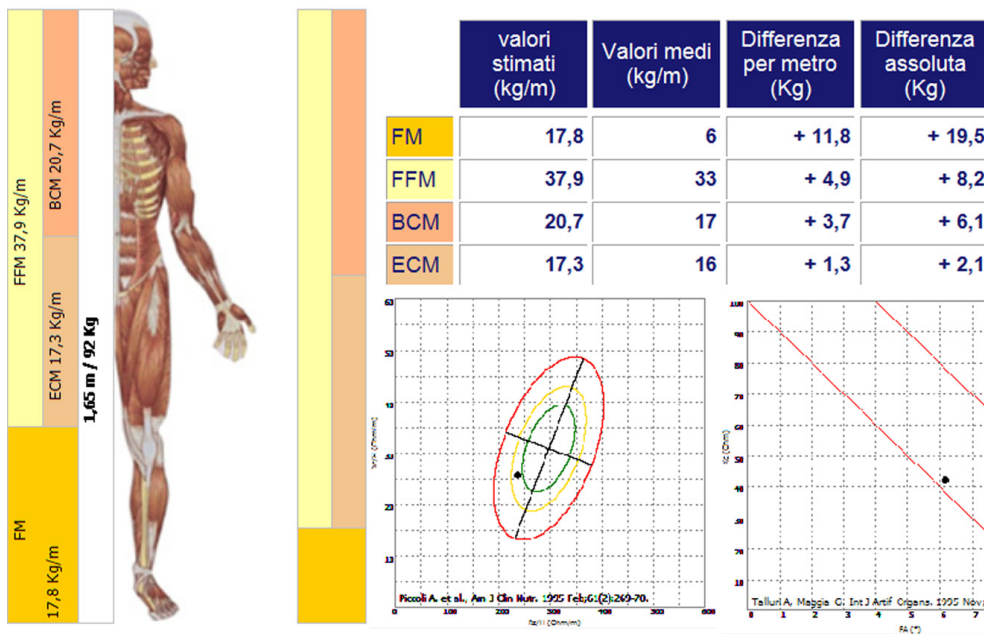

$(\mathrm{Kg})$
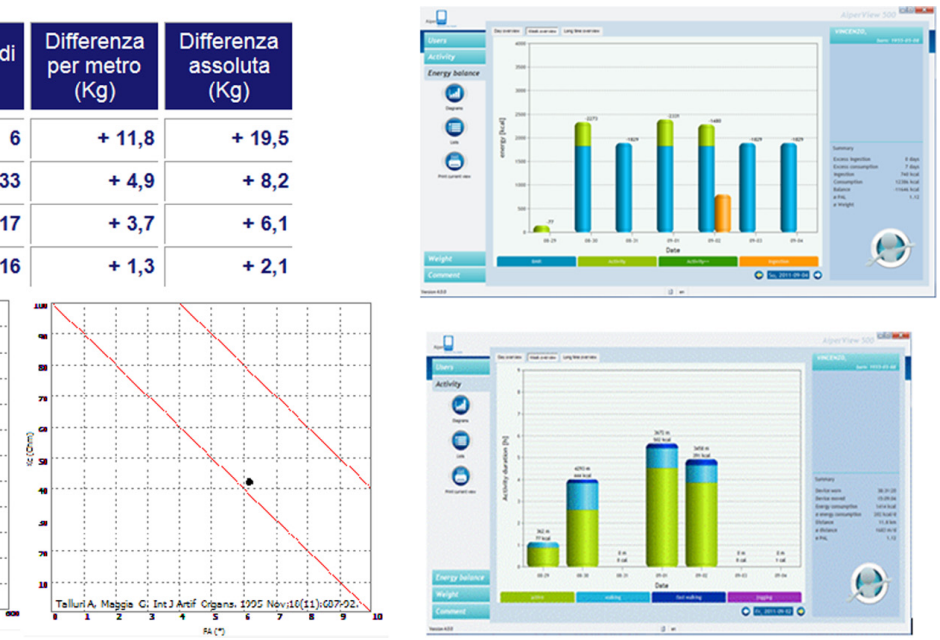

Figure 2 Accelerometer reports from software dedicated to lifestyle analysis.

appointment, along with flexibility on the sit-and-reach test. The handgrip test is most commonly used to assess muscular fitness. It is reported in the literature as in man, the force developed in this test, is an indicator related to the morbidity and to the life expectancy. The test is performed using a dynamometer handle, which is grasped separate times with the left hand and right hand for at least five seconds, whilst trying to develop maximum strength. The 30 -second chair stand test is normally performed by asking the patient to stand up and sit down in a chair as many times as possible during 30 seconds. The sit-and-reach test is carried out using a box in the shape of the table, on which the patient stretches and bends, and on which a scale is marked that allows us to estimate the point reached. This parameter can also be controlled for time. This test produces reasonably accurate and stable measurements of flexibility at the lower spine and hamstrings, which is often related to muscle pain and stiffness. $^{32}$

\section{Regular clinical follow-up}

Clinical follow-up will be carried out at approximately 4-week intervals to record anthropometric parameters and confirm participation in the program. This will include a clinical evaluation, an estimation of weight, body composition, and hydration (total body water, extracellular water, intracellular water, fat mass, fat-free mass), as well as lifestyle investigation using the accelerometer and questionnaire. If necessary, eating habits can be reinvestigated. For subjects taking part in this protocol, a follow-up 6MWT test will be performed approximately 3 months after initial treatment to assess cardiopulmonary performance, exercise tolerance, and the effects of any changes to the exercise program.
Periodic follow-up will also be an opportunity to obtain feedback from patients, and to check adherence and motivation to continue with the program.

Both the sports medicine and oncology units will meet at least twice a month to interpret and evaluate the data obtained. Routine follow-up at 4-week intervals includes a biomechanical assessment of muscle strength using the hand-grip test for the upper body and the 30 -second chair stand test for the lower body, as well as flexibility using the sit-and-reach test to check for gradual improvement in locomotor apparatus. It will also be possible to assess improvement in spontaneous physical activity, maintenance of weight within the normal range, exercise tolerance, and regaining of muscle tone and strength. Psychological status can also be assessed using a specific questionnaire. The oncology unit will also monitor plasma biomarkers for disease activity, normally related to progression of cancer, as an indicator of the effects of this "exercise prescription". A final summary report of each individualized exercise program will be written, which should provide valuable information for the patient and family physician.

\section{Summary}

This program incorporates general features which could be extended to include a wide range of cancer patients, not just those suffering from cancer of the breast or colon, though they are the most susceptible for their longest life expectancy. It is expected that the results of the program will show improvement in quality of life by gradual enhancement of spontaneous physical activity, as demonstrated by accelerometry, and progressive full participation in the exercise program could be anticipated. Depending on its application and use, this 
protocol could be useful for improving the management of cancer patients. The role of bioimpedance analysis will be important for verification of progressive improvement of water distribution in the body, with a progressive major component of the intracellular and therefore active compartment, rather than of one inactive or extracellular. The expectation of good adherence to this protocol is based on its ability to modulate the intensity of exercise according to exercise tolerance. It will be important to propose an exercise level appropriate to the clinical status of the individual patient. Another important issue is whether this exercise program will help to reduce the risk of cancer recurrence, given the known anti-inflammatory effects of exercise. Finally, the exercise program described here may have economic benefits to the health care system, but this will need investigation in the future.

\section{Disclosure}

The authors report no conflicts of interest in this work.

\section{References}

1. Peel JB, Sui X, Adams SA, Hébert JR, Hardin JW, Blair SN. A prospective study of cardiorespiratory fitness and breast cancer mortality. Med Sci Sports Exerc. 2009;41:742-748.

2. Schmitz KH, Courneya KS, Matthews C, et al. American College of Sports Medicine roundtable on exercise guidelines for cancer survivors. Med Sci Sports Exerc. 2011;42:1409-1426.

3. Kraus WE, Torgan CE, Duschan BD, et al. Studies of a targeted risk reduction intervention through defined exercise (STRIDE). Med Sci Sports Exerc. 2001;33:1774-1784.

4. Hainaut P, Plimoth A. Cancer as metabolic disease. Curr Opin Oncol. 2012;24:56-57.

5. Burnham TR, Wilcox A. Effects of exercise on physiological and psychological variables in cancer survivors. Med Sci Sports Exerc. 2002;34:1863-1867.

6. McTiernan A. Mechanisms linking physical activity with cancer. Nat Rev Cancer. 2008;8:205-211.

7. Dimeo F, Rumberg BG, Keul J. Aerobic exercise as therapy for cancer fatigue. Med Sci Sports Exerc. 1998;30:475-478.

8. Patterson RE, Flatt SW, Saquib N, et al. Medical comorbidities predict mortality in women with a history of early stage breast cancer. Breast Cancer Res Treat. 2010;122:859-865.

9. Mock V, Dow KH, Meares CJ, et al. Effects of exercise on fatigue, physical functioning, and emotional distress during radiation therapy for breast cancer. Oncol Nurs Forum. 1997;24:991-1000.

10. Stout NL, Binkley JM, Schmitz KH, et al. A prospective surveillance model for rehabilitation for women with breast cancer. Cancer. 2012;15:2191-2200.

11. Schwartz AL, Mori M, Gao R, Nail LM, King ME. Exercise reduces daily fatigue in women with breast cancer receiving chemotherapy. Med Sci Sports Exerc. 2001;33:718-723.

12. Campbell KL, Neil SE, Winters-Stone KM. Review of exercise studies in breast cancer survivors: attention to principles of exercise training. Br J Sports Med. 2012;46:909-916.

13. Haskell WL, Lee MI, Pate RR, et al. Physical Activity and Public Health: Updated Recommendation for Adults from the American College of Sports Medicine and the American Heart Association. Med Sci Sports Exerc. 2007.
14. Courneya KS. Exercise in cancer survivors: an overview of research. Med Sci Sports Exerc. 2003;35:1846-1852.

15. American College of Sports Medicine. Guidelines for Exercise Testing and Prescription, 8th ed. Philadelphia, PA: Lippincott Williams \& Wilkins; 2010.

16. Galvão DA, Newton RU. Review of exercise intervention studies in cancer patients. J Clin Oncol. 2005;23:899-909.

17. Speck RM, Courneya KS, Mâsse LC, Duval S, Schmitz KH. An update controlled physical activity trials in cancer survivors: a systematic review and meta analysis. J Cancer Surviv. 2010;4:87-100.

18. Maone A, Stefani L, Mascherini G, Galanti G. La prescrizione dell'esercizio fisico esperienza di un modello applicativo per la popolazione generale. [A model of exercise as prescription for the general population]. Medicina Dello Sport. 2011;64:351-364. Italian.

19. Coates AS, Fischer-Dillenbeck C, McNeil DR, et al. On the receiving end II. Linear analogue self-assessment (LASA) in evaluation of aspects of the quality of life of cancer patients receiving therapy. Eur J Clin Oncol. 1983;19:1633-1637.

20. American Thoracic Society Statement. Guidelines for the Six-Minute Walk Test. Am J Respir Crit Care Med. 2002;166:111-117.

21. Reis JP, Dubose KD, Ainsworth BE, Macera CA, Yore MM. Reliability and validity of the occupational physical activity questionnaire. Med Sci Sports Exerc. 2005;37:2075-2083.

22. Schag CC, Heinrich RL, Ganz PA. Karnofsky performance status revisited: reliability, validity, and guidelines. J Clin Oncology. 1984;2: 187-193.

23. Pollock ML, Gaesser GA, Butcher JD, et al. ACSM position stand: The Recommended Quantity and Quality of Exercise for Developing and Maintaining Fitness in Healthy Adults. Department of Health and Human Services. Physical Activity Guidelines for Americans Washington, DC: ODPHP Publication No U0036. Available from: http://www.health.gov/paguidelines/pdf/paguide.pdf. Accessed March 12, 2013.

24. Hsieh CC, Sprod LK, Carter SD, Hayward R, Schneider CM. Effects of treatment regimen on muscular fitness and quality of life in breast cancer survivors . Med Sci Sports Exerc. 2009;41:162.

25. Garber CE, Blissmer B, Deschenes MR, et al. Quantity and quality of exercise for developing and maintaining cardiorespiratory, musculoskeletal, and neuromotor fitness in apparently healthy adults: guidance for prescribing exercise. Position stand. Med Sci Sports Exerc. 2011;43:1334-1359.

26. Robertson RJ, Goss LF, Dubè J, et al. Validation of the adult OMNI scale of perceived exertion for cycle ergometer exercise. Med Sci Sports Exerc. 2004;36:102-108.

27. Hollen PJ, Gralla RJ. Measurement of quality of life in patients with lung cancer in multicenter trials of new therapies. Cancer. 1994;73: 2087-2098.

28. Swank AM. Exercise Physiology: Human Bioenergetics and its Application, 2nd ed. Med Sci Sports Exerc. 1996;28:1442-1443.

29. Schmitz KH, Courneya KS, Matthews C, et al. American College of Sports Medicine roundtable on exercise guidelines for cancer survivors. Med Sci Sports Exerc. 2011;42:1409-1426.

30. Owen N, Healy GN, Matthews CE, Dunstan DW. Too much sitting: the population health science of sedentary behaviour. Exerc Sport Sci Rev. 2010;38:105-113.

31. Gupta D, Lis GC, Dahlk SL, et al. The relationship between bioelectrical impedance phase angle and subjective global assessment in advanced colorectal cancer. Nutr J. 2008;7:19.

32. Jones CJ, Rikli RE, Max J, Noffal G. The reliability and validity of a chair sit-and-reach test as a measure of hamstring flexibility in older adults. Res Q Exerc Sport. 1998;69:338-343. 
International Journal of General Medicine

Dovepress

\section{Publish your work in this journal}

The International Journal of General Medicine is an international, peer-reviewed open-access journal that focuses on general and internal medicine, pathogenesis, epidemiology, diagnosis, monitoring and treatment protocols. The journal is characterized by the rapid reporting of reviews, original research and clinical studies across all disease areas.
A key focus is the elucidation of disease processes and management protocols resulting in improved outcomes for the patient. The manuscript management system is completely online and includes a very quick and fair peer-review system. Visit http://www.dovepress.com/ testimonials.php to read real quotes from published authors.

Submit your manuscript here: http://www.dovepress.com/international-journal-of-general-medicine-journal 